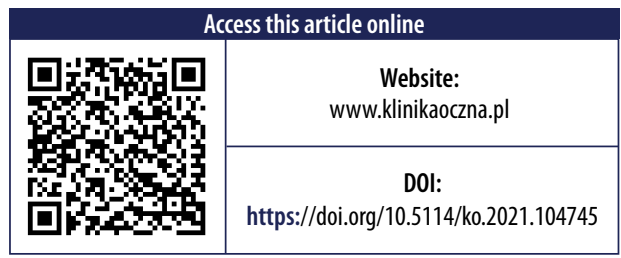

\title{
Modern methods of choroid imaging
}

\author{
Ewa Wasiluk, Iwona Obuchowska \\ Department of Ophthalmology, Medical University of Bialystok, Poland
}

\section{ABSTRACT}

The choroid is one of the most vascularized tissues in the body, responsible for over $70 \%$ of the blood flow in the eye. Choroid status seems to be a decisive factor in the pathogenesis of diseases such as age-related macular degeneration, central serous retinopathy, degenerative myopia, retinal vasculitis and some neoplastic diseases. However, traditional imaging methods, including indocyanine green angiography, ultrasound and optical coherence tomography (OCT), do not allow for the exact visualization of its structure. Until recently, the knowledge about choroid morphology was based mainly on post-mortem examinations. Only the application of novel modifications to the OCT technique, including enhanced depth imaging OCT (EDI-OCT), swept source OCT (SS-OCT) and OCT angiography, enabled its better visualization and examination. The increasing availability of these technologies enables fast, repeatable and non-invasive measurements of choroid thickness in everyday clinical practice. The correlation between new anatomical discoveries and functional results may make it possible to broaden the knowledge about pathogenesis of numerous eye diseases and even define new disease units.

KEY WORDS: choroidal imaging, choroidal anatomy, choroidal physiology, enhanced depth imaging optical coherence tomography, swept source optical coherence tomography, OCT angiography.

\section{ANATOMY AND PHYSIOLOGY OF THE CHOROID}

The choroid is the part of the uvea located in the posterior part of the eyeball, between the retina and the sclera. It is one of the most vascularized tissues in the body. The choroid is responsible for over $70 \%$ of all blood flow in the eye [1]. The amount of blood flowing in the choroid in the macular region is as much as seven times greater than in the peripheral part of the retina [1]. While the inner part of the retina (outside the macular region) is supplied by the vascular system originating from the central retinal artery, the vascular system of the retina's outer layers comes from the ciliary vessels of the uvea. The choroid provides nutrients and removes metabolic products from the external - the most functionally relevant - part of the retina, and the entire central part of the macula, the so-called foveal avascular zone (FAZ), covering an area of 250-600 $\mu \mathrm{m}$. The choroid also plays an important role in the process of vision itself. Its melanocytes absorb photons of light scattered inside the eyeball and the intense blood flow helps remove heat from the phototransduction metabolism.

The following structures can be distinguished in the histological structure of the choroid: Bruch's membrane adjacent to the retinal pigment epithelium (RPE) and three vascular layers: the inner layer of choriocapillaries, the middle Sattler's layer and the outer Haller's layer. The choriocapillaries are a dense network of capillaries, adjacent to Bruch's membrane. This layer is about $10 \mu \mathrm{m}$ thick in the fovea, where the density of the vessels is the highest, and about $7 \mu \mathrm{m}$ thick at the edges of the macula. The choriocapillary endothelium has numerous fenestrations, especially on the retinal side, which facilitates the directional flow of oxygen and nutrients from the choroid to the RPE [2]. Their high permeability to proteins also contributes to the formation of high oncotic pressure in the extravascular stroma, which promotes the flow of substances from the retina to the choroid. Such fenestrations were not found in the endothelium of larger choroidal vessels. The polarity of the choriocapillary layer is also clear with respect to the expression of vascular endothelial growth factor (VEGF) receptors. The VEGF-1 and VEGF-2 receptors are mainly located in the vicinity of Bruch's membrane. Below the choriocapillary layer, there is an area of medium and small arteries, belonging to Sattler's layer, followed by large blood vessels of Haller's layer. The extravascular cavity contains collagen and elastic fibers, fibroblasts, smooth muscle cells and numerous, very large melanocytes. As in other types of connective tissue, mast cells, macrophages, lymphocytes and plasmocytes are also found here. From the outside, the cho- 
roid is adjacent to the suprachoroidal space, which is the transition zone between it and the sclera. This area contains large, free spaces lined with endothelium, called lacunes and collagen fibers, fibroblasts and melanocytes.

The regulation of blood flow in the choroid is controlled by the autonomic nervous system, both sympathetic and parasympathetic [3]. However, there is no typical autoregulation dependent on cellular metabolism. As a consequence, systemic hypoxia or increased intraocular pressure may result in a decrease in oxygen partial pressure in the choroid circulation. Lack of self-regulation, reacting to these changes, may lead to hypoxia, very harmful to the proper activity and survival of photoreceptors.

\section{CHOROID IMAGING METHODS}

The choroid plays a very important role in the pathogenesis of various chorioretinal diseases such as age-related macular degeneration (AMD), central serous chorioretinopathy (CSC), or degenerative myopia, retinal vasculitis and some neoplastic diseases. Choroid blood flow disorders may also be an underlying cause of glaucoma and diabetic retinopathy [4]. Quantitative assessment of the choroid has been quite challenging with traditional imaging modalities such as indocyanine green angiography (ICGA) and ultrasonography due to limited resolution and repeatability. With the advent of new technology, such as optical coherence tomography (OCT) or OCT angiography (OCTA), detailed visualization of the choroid in vivo is now possible.

\section{OLDER METHODS OF CHOROID ASSESSMENT}

Before modern diagnostic methods were developed, the assessment of a choroid was possible either in vitro by histological examinations or in vivo by ICGA, laser Doppler flowmetry or standard ultrasonography [5].

The histological assessment of a choroid has limited clinical use, by the mere fact that the examination is performed post-mortem. ICGA uses a dye called indocyanine green, which shows higher affinity binding with plasma proteins than fluorescein. Large conglomerates of proteins and dye remain in the vascular system, because they are unable to pass through small choriocapillaris fenestrations. The main advantage of indocyanine is that its excitation wavelength is in the near-infrared range (790-805 nm). Light of this frequency can penetrate not only through the retinal layers, including the RPE, but also through fluid, lipid and hemorrhage, allowing the ICGA to produce images deeper than in fluorescein angiography (FA). This makes it possible to visualize choroid vessels, as well as pathologies such as choroidal neovascularization (CNV) and choroidal polyps [6].

While ICGA is an invasive test, laser Doppler flowmetry is a non-invasive diagnostic method that allows the evaluation of hemodynamic parameters of subfoveal choroidal flow by determining the average speed and number of erythrocytes moving in a specific volume. Laser Doppler flowmetry has been used to show that choroidal circulation is decreased in such diseases as $A M D$, diabetic retinopathy, and retinitis pigmentosa $[7,8]$.
Ultrasonography is an important diagnostic tool when the assessment of the posterior pole of the eyeball is not possible due to opaque optical media. In particular, it enabled the detection of thickening and tumors in the choroid. However, because of its low image resolution, detecting small changes in the choroid is difficult, and it is not an ideal method for measuring a relatively thin tissue such as the choroid [9].

Although these three techniques were used for many years to detect normal and pathological choroidal vessel and choroidal circulation changes, none of them provided in vivo cross-sectional images of the anatomy of the RPE and choroidal layers, and none provided sufficient data regarding true choroidal thickness and morphology. This became possible only after introducing new imaging methods into clinical practice, as described below.

\section{OPTICAL COHERENCE TOMOGRAPHY}

Optical coherence tomography is a relatively new method of imaging, which has enabled the acquisition of cross-sectional images of the retina and choroid. The OCT examination thus provides information on the depth at which the lesion is located. This method is based on the analysis of the light reflection coefficient from different layers of eye tissues, mainly retina, allowing one to obtain its cross-sections with very high resolution. During the examination, the light beam scans the retina at different depths, and the resulting multiple single axial scans, called A-scans, are recorded as a single image (tomogram), called a B-scan. In this aspect, OCT imaging is somewhat analogous to ultrasound imaging, except it uses light instead of sound, and the optical echo technique has a higher resolution and does not require contact with the examined tissue [10]. Importantly, as the depth of the imaged tissue increases, the image resolution and intensity of the received signal decrease.

The first OCT technology was time domain OCT (TD-OCT), which was soon replaced by spectral domain OCT (SD-OCT), a newer generation OCT method commonly used these days. In this technology, in order to obtain an image, the light reflected from the various tissue layers is detected simultaneously by a high-speed spectrometer and processed with a Fourier transform. For this reason, SD-OCT is also known as Fourier domain OCT. The use of a spectrometer to detect light reflected from tissue allows SD-OCT instruments to acquire 20000-52 $000 \mathrm{~A}$-scans per second and produce images with $5 \mu \mathrm{m}$ resolution [12].

Despite its numerous advantages, conventional OCT imaging has its limitations, especially when it comes to choroid testing. This is because the near-infrared light source used $(800 \mathrm{~nm})$ is scattered by hyperreflective RPE, resulting in a very weak signal reflected from the choroid. Only the latest SD-OCT devices using 830-855 nm wavelength (Xephilio OCT-A1, Canon Medical Systems, USA; Revo NX 130, Optopol Technology Ltd., Poland) with the appropriate change of focus allow visualization of the choroidoscleral junction. 


\section{NEW MODIFICATIONS TO THE OPTICAL COHERENCE TOMOGRAPHY TECHNIQUE}

New diagnostic possibilities opened up in 2008, when a new imaging method called enhanced depth imaging OCT was introduced [13]. This technique is based on the fact that the result of using the Fourier transform to decode the interferometer signal is in fact two coupled images, which are mirror reflections. According to the applied convention, only one of the images is displayed in commercial OCT systems. The inner layers of the retina are directed upwards on the screen and have the highest resolution, while the deeper layers at the bottom of the display are presented with less accuracy. An inverted mirror image is not presented $[13,14]$. By shifting the top of the imaging sensitivity backwards by concentrating the largest part of the light beam at the level of the choroid and sclera, these structures can be imaged more accurately. Although the wavelength used in this study is not optimal for choroidal imaging, the new technique has allowed for an additional 500-800 $\mu \mathrm{m}$ light penetration into the eye structures [2]. The effect of shifting the peak of sensitivity deep into the eyeball for better visualization of the choroid is a decrease in the imaging accuracy of the vitreous body. In SD-OCT, one of the imaging modes can be selected: the conventional scan, which visualizes the vitreous body and retina
(Figure 1A), and the EDI mode, which displays the retina and the choroid (Figure 1B). In most cases, it is not possible for both extreme structures (vitreous body and choroid) to be optimally visible at the same time [13].

Another improvement in choroidal imaging was the introduction of swept source optical coherence tomography (SS-OCT) using tunable lasers as a light source. In SS-OCT, the laser emits successively single waves at different frequencies, and their reflections are instantly recorded by the photodetector. The use of infrared wavelengths (> $1000 \mathrm{~nm}$ ) provides better penetration into the eye tissue than red light used in SD-OCT devices, eliminating obscuring artifacts overlapping e.g. from blood vessels [15]. It is also easier to penetrate through advanced lens opacities and hemorrhage. In addition, the abandonment of the SD-OCT's sensitivity-limiting spectrometer in favor of the photodetector has enabled the simultaneous obtaining of high-quality image of both the choroid and the vitreous body (Figure 2) [16]. The new technology has also allowed for an increase in imaging speed, which is particularly important when examining elderly people and patients with low visual acuity that prevents correct fixation.

However, the use of longer light waves is also associated with certain limitations. The resolution and quality of the images obtained with SS-OCT compared to SD-OCT are re-
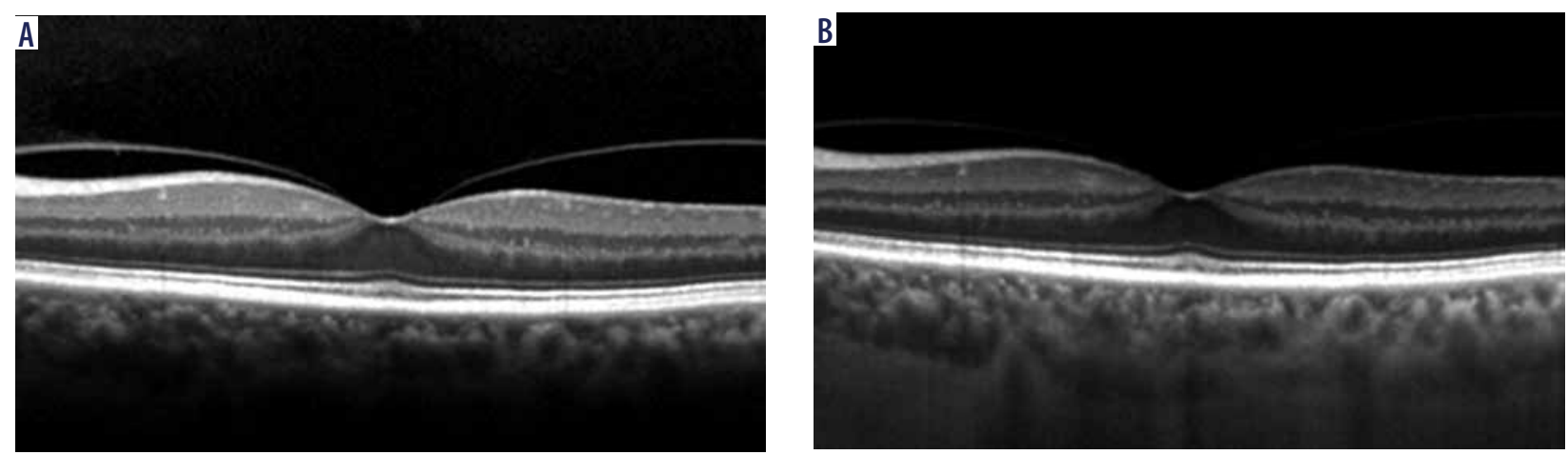

Figure 1. Enhanced depth imaging OCT: A) conventional scan; the sensitivity peak is located at the posterior border of the vitreous body; B) the 0CT device is closer to the eye to reverse the image and direct the sensitivity peak to the inner layer of the sclera

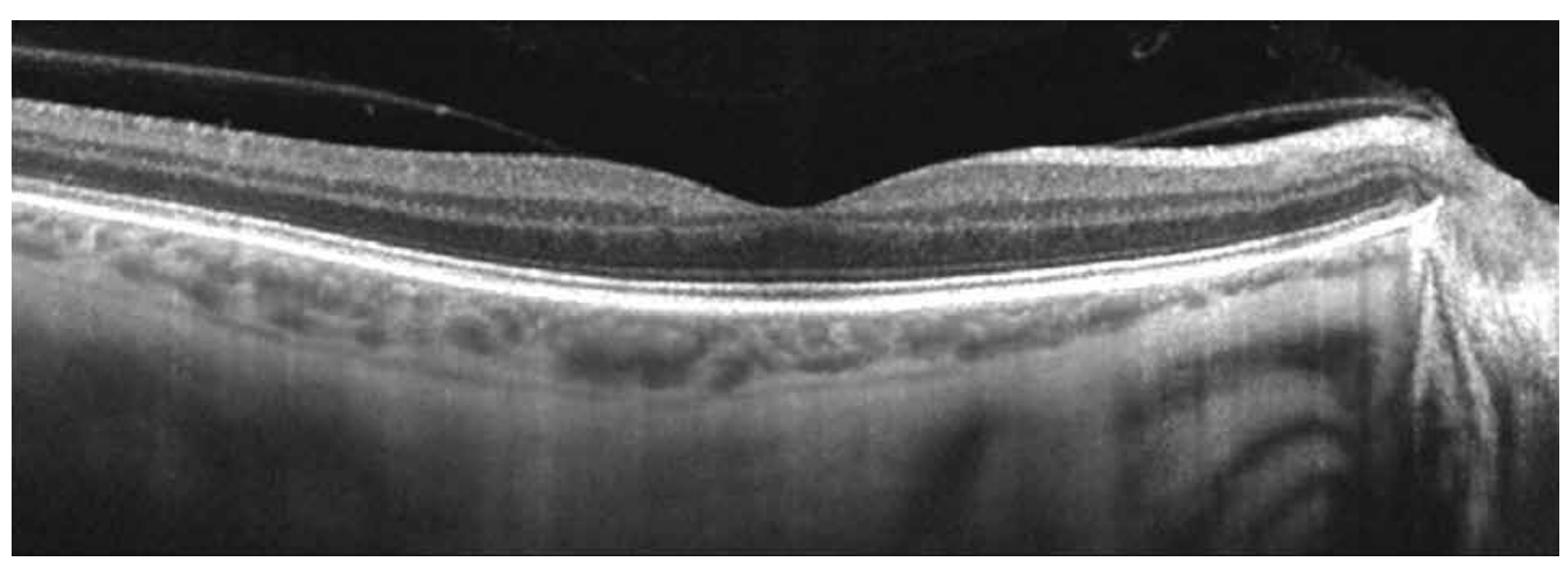

Figure 2. Retina image obtained using SS-OCT technique. Both the posterior border of the vitreous body and the choroid are clearly visible on the same scan 
duced $[17,18]$. In addition, longer light waves are more easily absorbed by water. Since it is the main component of the vitreous body, this limits the possibility of further extension of the wavelength in the imaging of the eye with SS-OCT.

Evaluation of the choroid is based mainly on the determination of its thickness. Using the SD-OCT and SS-OCT techniques, regardless of the device being used, the examination of the choroidal thickness is performed manually by the examiner, by marking the external RPE and internal sclera boundary. Digital calipers are placed on the outer edge of the hyper-reflective RPE line and the inner border of the hyper-reflective surface behind the large vessel layer of the choroid (Figure 3 ). The line connecting these points should run perpendicular to the RPE line. The measurement point is usually the area under the geometric center of the fovea. Numerous studies have confirmed very high repeatability of manual measurements of choroid thickness, even if performed by different people, on different instruments and at different times. The automatic method of measurement, using automated retinal and choroidal segmentation, although it has the advantages of being much faster and does not require a high level of examiner's skill, is also burdened with a high coefficient of measurement variation between successive tests, as well as between measurements made on different devices. Segmentation errors require manual correction. It is also not possible to replace the individual approach to individual variability of the subject.

\section{CHOROIDAL THICKNESS}

The thickness of the choroid is an important parameter that affects the proper activity of the retina. Normal choroidal thickness was first described by Margolis and Spaide [14] using the Spectralis (Heidelberg Engineering, Heidelberg, Germany) and Manjunath et al. [19] using the Cirrus HD-OCT (Carl Zeiss Meditec, Dublin, CA, USA). A lot is already known about this. The choroid is not the same over the entire surface. In healthy eyes, it is usually thickest under the fovea, with an average of $262 \mu \mathrm{m}$ to $354 \mu \mathrm{m}$ [14, 19-21]. Within the macula, the choroid is usually thicker in the upper and the lower parts, compared to the temporal and nasal parts [20]. At the periphery, its thickness is greatest in the upper quadrant, followed by the lower, temporal and nasal quadrants, where it rapidly decreases towards the optic nerve [14, 20-22]. Thinning of the uvea in the inferior quadrant corresponds to the location of the embryonic visual gap and may represent the "relative coloboma" [20, 23]. In addition, thinning of the choroid between the fovea and the optic nerve disc may predispose to peripapillary atrophy [22]. In the vicinity of the optic nerve, the choroid is the thinnest in the lower part, which may contribute to development of glaucoma neuropathy in susceptible individuals [13, 22, 23].

Many factors influence the thickness of the choroid. The most important one is age [20]. In people who are over 60 years old, the thickness of the choroid gradually decreases by $4-5 \mu \mathrm{m}$ every year $[21,24]$. Although there are large individual differences, the average subfoveal choroidal thick-

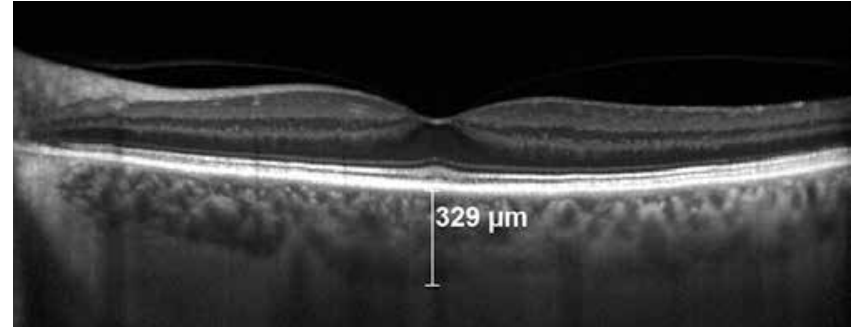

Figure 3. Measurement of subfoveal choroidal thickness using a manual caliper on the EDI-OCT scan

ness in individuals over 60 years of age is $197 \mu \mathrm{m}[20,21]$. In the case of younger people, age has little effect on choroid thickness [22]. Age-related choroidal thinning is likely to be accompanied by a reduction in the supply of oxygen and metabolites to RPE and photoreceptors, which may play a role in the development of retinal degenerative diseases [14, $21,22]$. The thickness of the choroid also varies according to gender. On average, healthy men have $7 \%$ more choroid volume, which may explain the higher incidence of AMD among women, who already have a thinner choroid $[22,24]$. There is a positive correlation between the subfoveal thickness of the choroid and the best corrected visual acuity, which confirms the functional dependence of photoreceptors on choroid support $[24,25]$.

Other important factors influencing the thickness of the choroid are eye refraction and axial length of the eyeball $[20,24]$. There is a negative correlation between the thickness of the choroid and the degree of myopia [20, 21, 24]. In other words, the thickness of the choroid decreases in myopia as the refractive error increases. Similarly, an increase in the axial length of the eyeball is associated with a decrease in the thickness of the choroid $[20,22]$. In those eyes where myopia is greater than -1.0 diopters, the predicted decrease in subfoveal choroidal thickness is approximately $15 \mu \mathrm{m}$ for each additional diopter of visual impairment, and $32 \mu \mathrm{m}$ for each $1 \mathrm{~mm}$ of increase in the axial length of the eyeball [24]. Other biometric indicators of the eyeball that accompany the reduced thickness of the choroid are a shallower anterior chamber, a thinner lens and a steeper cornea [24]. Interestingly, there was no confirmed correlation between the thickness of the choroid and hyperopia or retinal thickness [19, 23, 24].

Choroid thickness changes during the day and night due to daily fluctuations in choroidal blood flow [26, 27]. A 24-hour choroidal examination indicates that it is the thickest between 3.00 and 9.00 am and the thinnest between 3.00 and $9.00 \mathrm{pm}$ [26]. Horizontal position at night causes gravitational increase of venous hydrostatic pressure, which results in edema of the choroid veins and increase of its volume [28]. A correlation between intraocular pressure and choroid thickness was not confirmed [26].

Using modern OCT testing techniques, it is not possible to depict Bruch's membrane, which lies immediately under the RPE. The echoes from this tissue cannot be differentiated from the strong signals coming from the RPE. However, in eyes where the RPE has separated from Bruch's membrane as 
in AMD or CSC, Bruch's membrane appears as a thin line of mild hyperreflectivity [5]. Also, choriocapillaries cannot be visualized using current OCT technology. However, the next layer contains mid-sized vessels (Sattler's layer), which appear as two to four rows of small hyperreflective spots immediately beneath the hyperreflective line of the RPE. This layer usually appears as the first 20-30 microns of choroid under the RPE $[5,29]$. The largest vessels, also called Haller's layer, can be distinguished as round or oval shapes deeper in the choroid.

\section{OPTICAL COHERENCE TOMOGRAPHY ANGIOGRAPHY}

Given the commonly used diagnostic methods such as angiography or OCT, varying in terms of utility, there was an urgent need to create a research technique that combines the advantages of imaging of the anatomy and a kind of tissue histology (OCT) with vascular imaging (angiography, Doppler). OCT-based angiography (OCT-A), a novel, non-invasive method of flow imaging in retinal and choroidal vessels, has thus become a reality. OCTA uses the phenomenon of laser reflection from the surfaces of moving red blood cells, creating a detailed image of the retina and choroid vascular system in the fovea. It enables the assessment of the histological structure of the retina - superficial plexus (vessels in the nerve fiber layer), deep plexus (vessels at the level of the inner nuclear layer and outer plexiform layer), external retina and choriocapillaries - and allows one to visualize vascular pathologies in all these layers (Figure 4).

Compared to conventional angiography methods, OCT-A is a much quicker, completely non-invasive test, without the use of contrast, the leakage of which may mask the details of the image. The OCT-based approach provides 3-D imaging information of the macula and, in addition to the qualitative analysis done on standard angiography, may allow quantitative analysis, since it provides numerical data about flow area and flow density maps (e.g. Optovue AngioVue, Optovue, Inc., Freemont, CA).

As a light source dependent device, OCT-A technology is affected by different types of artifacts. Media opacities, pupil vignetting, and defocusing of the light beam can lead to signal attenuation and shadowing artifacts [30]. Furthermore, abnormalities in deeper vessels of the retina may be obscured by more superficial blood vessels (projection artifacts) [30]. OCT-A examination is also extremely motion-sensitive and requires good patient cooperation [31]. In addition, automated segmentation in pathological conditions is not always reliable and often requires manual editing of layers for correct interpretation [32]). Another important limitation is the limited area of visualization of current commercial OCT-A systems $\left(3 \mathrm{~mm}^{2}\right.$ to $12 \mathrm{~mm}^{2}$ ) [31]. For now, as the scanning area increases, the resolution drops significantly in order to maintain an acceptable scanning time. Lastly, OCT-A technology is directed at "motion" detecting, and therefore low amounts of blood flow near the threshold of the device may not be detected [30].

The correct interpretation of the obtained images may be significantly hampered by the above-mentioned limitations. OCT-A of the choriocapillaris is particularly susceptible to artifacts. Areas of low OCT-A signal, especially likely to appear below the RPE due to its melanin content, may be caused by low flow, low OCT signal, or both. Due to the limited resolution, the segmented choriocapillaris slab may also include vessels from adjacent choroidal layers. To avoid misinterpretation, it is necessary to evaluate OCTA images in conjunction with en face and cross-sectional OCT images segmented at the level of choriocapillaris.

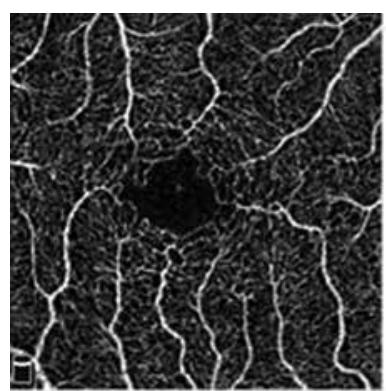

ngos oct - Supertical

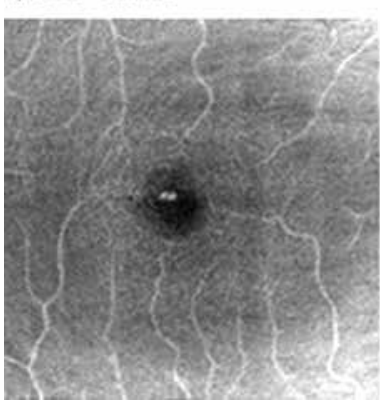

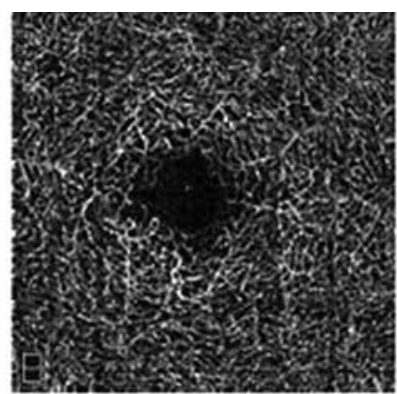

Nopo/OCT - Desp

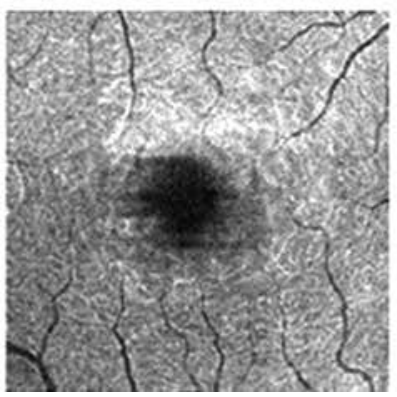

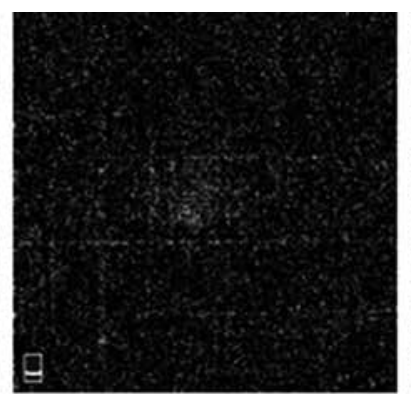

Nego / OCT - Ouser Rotna

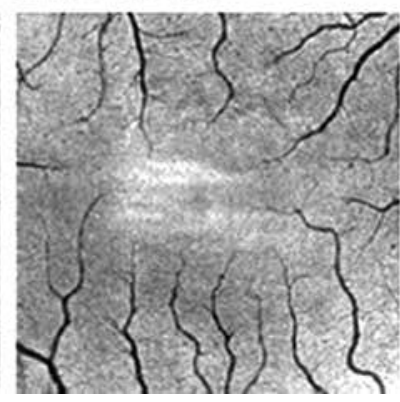

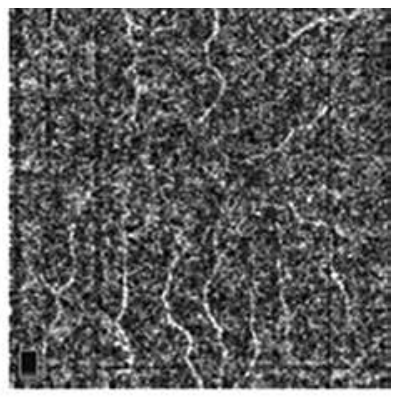

Ango / OCT - Choreds Captory

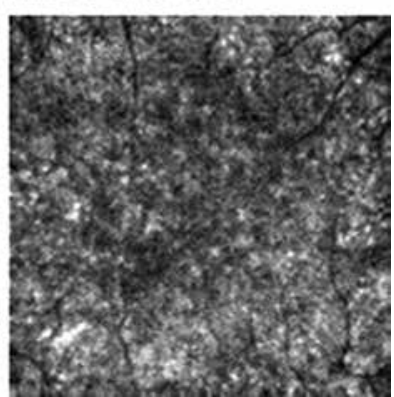

Figure 4. Optical coherence tomography based angiography. From left to right: superficial plexus image, deep plexus image, external retina image, choriocapillary layer image 


\section{MODERN IMAGING METHODS IN CLINICAL PRACTICE}

The introduction of novel choroidal imaging techniques allowed the distinction of numerous completely new disease entities and clinical anomalies.

The concept of pachychoroid diseases (Greek: pachy, thick) has been established over the past few years based on clinical findings obtained through the use of EDI-OCT and SS-OCT in eyes with AMD and CSC [33]. Recent studies have finally provided definitive genetic confirmation of this concept [34]. The spectrum of pachychoroid disorders includes the following: CSC, pachychoroid pigment epitheliopathy, pachychoroid neovasculopathy, and pachychoroid geographic atrophy. Previous studies have also suggested the distinction of two subtypes of polypoidal choroidal vasculopathy (PCV): pachychoroid PCV and drusen-driven PCV [35]. Although clinical manifestations of these entities vary considerably, they share some crucial morphological findings in the choroid: increased thickness and dilated outer choroidal vessels (pachyvessels) with obliteration of the choriocapillaris and Sattler's layer noted in structural OCT, as well as choroidal vascular hyperpermeability on ICGA [36]. It appears that the presence of pachychoroid phenotype may affect the response to anti-VEGF therapy and patient prognosis $[37,38]$. However, further research is necessary to understand its true nature and improve treatment outcomes.

In turn, age-related choroidal atrophy (ARCA) is characterized by significant thinning of the choroid. EDI-OCT shows the loss of choroidal vessels with a smaller diameter, so that the remaining larger vessels fill its entire cross section [39]. These changes exceed age-dependent physiological changes and despite the pigmentation abnormalities in the posterior pole that mimic AMD, ARCA and AMD are two different entities.

Furthermore, modern methods of choroid imaging are also proving to be useful in understanding many of the longknown retinal conditions. There are multiple reports of choroidal thickness measurements using EDI-OCT and SS-OCT during anti-VEGF treatment in a number of chorioretinal diseases (AMD, PCV, CSC, diabetic macular edema) [40-43]. In the case of exudative AMD, these measurements may be of significance as a potential prognostic factor of the disease course [44], as well as an indicator of CNV activity [45]. In addition, OCTA enables precise assessment of the morphology and extent of the neovascular membrane in the exudative AMD or secondary to other causes (e.g. pathologic myopia, CSC, uveitis), as well as its changes after intravitreal injections [31]. Moreover, it has the potential for detection of neovascular complexes in non-exudative cases of AMD, which would be difficult to detect using SD-OCT or FA, and thus contributes to a more effective and closer follow-up [31].

Assessment of choriocapillaris flow is used in dry AMD to determine the extent of atrophy and choroidal vasculature changes [46]. High hopes are also associated with the use of OCT-A for the quantification of the FAZ and non-perfused areas in the early diagnosis of diabetic patients with minimal or no diabetic retinopathy [31].

Choroidal visualization has turned out to be a useful way to assess the severity of the inflammation in the posterior pole and a non-invasive method of treatment response monitoring. Transiently increased choroidal thickness has been reported in the acute stages of several posterior uveitis such as Vogt-Koyanagi-Harada disease, multiple evanescent white dot syndrome and multifocal choroiditis [47]. OCT-A is emerging as a particularly valuable tool in distinguishing between active inflammatory lesions, active vascular lesions and inactive fibrotic lesions, and potentially improving our understanding of the pathophysiology of these diseases as well as their follow-up and management [48].

\section{CONCLUSIONS}

The new choroid imaging methods described above have proven to be very useful in the imaging of chorioretinal pathology, both in clinical use and in improving our understanding of the pathogenesis and evolution of retinal diseases. Their further in vivo applications are still being investigated. Although technological development is still ongoing, thanks to the increasing availability, utility and non-invasive nature, EDI-OCT, SS-OCT and OCT-A have already become important tools in standard eye care.

\section{DISCLOSURE}

The authors declare no conflict of interest.

\section{References}

1. Parver LM, Auker C, Carpenter D0. Choroidal blood flow as a heat dissipating mechanism in the macula. Am J Ophthalmol 1980;89: 641-646.

2. Mrejen S, Spaide RF. Optical coherence tomography: imaging of the choroid and beyond. Surv Ophthalmol 2013; 58: 387-429.

3. Scholfield CN, McGeown JG, Curtis TM. Cellular physiology of retinal and choroidal arteriolar smooth muscle cells. Microcirculation 2007; 14: 11-24.

4. Unsal E, Eltutar K, Zirtiloglu S, et al. Choroidal thickness in patients with diabetic retinopathy. Clin Ophthalmol 2014; 8: 637-642.

5. Sezer T, Altinisik M, Koytak IA, Ozdemir MH. The Choroid and Optical Coherence Tomography. Turk J Ophthalmol 2016; 46: 30-37.

6. Yannuzzi LA. Indocyanine green angiography: a perspective on use in the clinical setting. Am J Ophthalmol 2011; 151:745-751 e1.

7. Gugleta K, Orgul S, Flammer I, et al. Reliability of confocal choroidal laser Doppler flowmetry. Invest Ophthalmol Vis Sci 2002; 43: 723-728.

8. Pemp B, Schmetterer L. Ocular blood flow in diabetes and age-related macular degeneration. Can J Ophthalmol 2008; 43: 295-301.

9. Malhotra A, Minja FJ, Crum A, Burrowes D. Ocular anatomy and cross-sectional imaging of the eye. Semin Ultrasound CT MR 2011; 32: $2-13$. 
10. Wong IY, Koizumi H, Lai WW. Enhanced depth imaging optical coherence tomography. Ophthalmic Surg Lasers Imaging 2011; 42 Suppl: S75-84.

11. Sull AC, Vuong LN, Price LL, et al. Comparison of spectral/Fourier domain optical coherence tomography instruments for assessment of normal macular thickness. Retina 2010; 30: 235-245.

12. Adhi M, Duker JS. Optical coherence tomography - current and future applications. Curr Opin Ophthalmol 2013; 24: 213-221.

13. Spaide RF, Koizumi H, Pozzoni MC. Enhanced depth imaging spectral-domain optical coherence tomography. Am J Ophthalmol 2008; 146: 496-500.

14. Margolis R, Spaide RF. A pilot study of enhanced depth imaging optical coherence tomography of the choroid in normal eyes. Am J Ophthalmol 2009; 147: 811-815.

15. Singh SR, Vupparaboina KK, Goud A, et al. Choroidal imaging biomarkers. Surv Ophthalmol 2019; 64: 312-333.

16. Adhi M, Liu JJ, Qavi AH, et al. Choroidal analysis in healthy eyes using swept-source optical coherence tomography compared to spectral domain optical coherence tomography. Am J Ophthalmol 2014; 157: 1272-1281 e1.

17. Yun S, Tearney G, Bouma B, et al. High-speed spectral-domain optical coherence tomography at 1.3 mum wavelength. Opt Express 2003; 11:3598-3604.

18. de Boer JF, Cense B, Park BH, et al. Improved signal-to-noise ratio in spectral-domain compared with time-domain optical coherence tomography. Opt Lett 2003; 28: 2067-2069.

19. Manjunath V, Taha M, Fujimoto JG, Duker JS. Choroidal thickness in normal eyes measured using Cirrus HD optical coherence tomography. Am J Ophthalmol 2010; 150: 325-329 e1.

20. Ikuno Y, Kawaguchi K, Nouchi T, Yasuno Y. Choroidal thickness in healthy Japanese subjects. Invest Ophthalmol Vis Sci 2010; 51: 2173-2176.

21. Ding X, Li J, Zeng J, et al. Choroidal thickness in healthy Chinese subjects. Invest Ophthalmol Vis Sci 2011; 52: 9555-9560.

22. Barteselli $G$, Chhablani J, El-Emam S, et al. Choroidal volume variations with age, axial length, and sex in healthy subjects: a threedimensional analysis. Ophthalmology 2012; 119: 2572-2578.

23. Ho J, Branchini L, Regatieri C, et al. Analysis of normal peripapillary choroidal thickness via spectral domain optical coherence tomography. Ophthalmology 2011; 118: 2001-2007.

24. Wei WB, Xu L, Jonas JB, et al. Subfoveal choroidal thickness: the Beijing Eye Study. Ophthalmology 2013; 120: 175-180.

25. Lee JY, Lee DH, Lee JY, Yoon YH. Correlation between subfoveal choroidal thickness and the severity or progression of nonexudative age-related macular degeneration. Invest Ophthalmol Vis Sci 2013; 54: 7812-7818.

26. Usui $S$, Ikuno $Y$, Akiba $M$, et al. Circadian changes in subfoveal choroidal thickness and the relationship with circulatory factors in healthy subjects. Invest Ophthalmol Vis Sci 2012; 53: 2300-2307.

27. Tan CS, Ouyang Y, Ruiz H, Sadda SR. Diurnal variation of choroidal thickness in normal, healthy subjects measured by spectral domain optical coherence tomography. Invest Ophthalmol Vis Sci 2012; 53: 261-266.

28. Shinojima A, Iwasaki K, Aoki K, et al. Subfoveal choroidal thickness and foveal retinal thickness during head-down tilt. Aviat Space Environ Med 2012; 83: 388-393.

29. Branchini LA, Adhi M, Regatieri CV, et al. Analysis of choroidal morphologic features and vasculature in healthy eyes using spectral -domain optical coherence tomography. Ophthalmology 2013; 120: 1901-1908.

30. Spaide RF, Fujimoto JG, Waheed NK. Image Artifacts in Optical Coherence Tomography Angiography. Retina 2015; 35: 2163-2180.

31. De Oliveira PR, Berger AR, Chow DR. Optical coherence tomography angiography in chorioretinal disorders. Can J Ophthalmol 2017; 52: 125-136.

32. Chen FK, Viljoen RD, Bukowska DM. Classification of image artefacts in optical coherence tomography angiography of the choroid in macular diseases. Clin Exp Ophthalmol 2016; 44: 388-399.

33. Imamura Y, Fujiwara T, Margolis R, Spaide RF. Enhanced depth imaging optical coherence tomography of the choroid in central serous chorioretinopathy. Retina 2009; 29: 1469-1473.

34. Yamashiro K, Hosoda Y, Miyake M, et al. Characteristics of pachychoroid diseases and age-related macular degeneration: multimodal imaging and genetic backgrounds. J Clin Med 2020; 9: 2034.

35. Liu ZY, Li B, Xia S, Chen YX. Analysis of choroidal morphology and comparison of imaging findings of subtypes of polypoidal choroidal vasculopathy: a new classification system. Int J Ophthalmol 2020; 13: 731-736.

36. Dansingani KK, Balaratnasingam C, Naysan J, Freund KB. En face imaging of pachychoroid spectrum disorders with swept-source optical coherence tomography. Retina 2016; 36: 499-516.

37. Chang YC, Cheng CK. Difference between pachychoroid and nonpachychoroid polypoidal choroidal vasculopathy and their response to anti-vascular endothelial growth factor therapy. Retina 2020; 40: 1403-1411.

38. Jung BJ, Kim JY, Lee JH, et al. Intravitreal aflibercept and ranibizumab for pachychoroid neovasculopathy. Sci Rep 2019; 9: 2055.

39. Spaide RF. Age-related choroidal atrophy. Am J Ophthalmol 2009; 147: 801-810.

40. Ting DSW, Yanagi Y, Agrawal R, et al. Choroidal remodeling in age-related macular degeneration and polypoidal choroidal vasculopathy: a 12-month prospective study. Sci Rep 2017; 7: 7868.

41. Rayess N, Rahimy E, Ying GS, et al. Baseline choroidal thickness as a predictor for response to anti-vascular endothelial growth factor therapy in diabetic macular edema. Am J Ophthalmol 2015; 159: 85-91 e1-3.

42. Jung BJ, Lee K, Park JH, Lee JH. Chorioretinal response to intravitreal aflibercept injection in acute central serous chorioretinopathy. Int J Ophthalmol 2019; 12: 1865-1871.

43. Jamiołkowska I, Figurska M, Rękas M. Zmiany grubości naczyniówki u chorych na wysiękową postać zwyrodnienia plamki związanego z wiekiem leczonych afliberceptem w obserwacji rocznej. Klinika Oczna 2019; 121 (3).

44. Kang HM, Kwon HJ, Yi JH, et al. Subfoveal choroidal thickness as a potential predictor of visual outcome and treatment response after intravitreal ranibizumab injections for typical exudative age-related macular degeneration. Am J Ophthalmol 2014; 157: 1013-1021.

45. Invernizzi A, Benatti E, Cozzi M, et al. Choroidal Structural Changes Correlate With Neovascular Activity in Neovascular Age Related Macular Degeneration. Invest Ophthalmol Vis Sci 2018; 59: 3836-3841.

46. Waheed NK, Moult EM, Fujimoto JG, Rosenfeld PJ. Optical coherence tomography angiography of dry age-related macular degeneration. Dev Ophthalmol 2016; 56: 91-100.

47. Pichi F, Sarraf D, Arepalli S, et al. The application of optical coherence tomography angiography in uveitis and inflammatory eye diseases. Prog Retin Eye Res 2017; 59: 178-201.

48. Marchese A, Agarwal A, Moretti AG, et al. Advances in imaging of uveitis. Ther Adv Ophthalmol 2020; 12: 2515841420917781. 